\title{
Solving Schrodinger equation specializing to the Stark effect in linear potential by the canonical function method
}

\author{
L. Farhang Matin $\cdot$ H. Hasan Bouzari • \\ F. Ahmadi
}

Received: 24 March 2014/ Accepted: 10 June 2014/Published online: 1 July 2014

(C) The Author(s) 2014. This article is published with open access at Springerlink.com

\begin{abstract}
The canonical function method (CFM) is a powerful accurate and fast method that solves the Schrodinger equation for the eigenvalues directly without evaluating the eigenfunctions. In this work, it is applied to the solution 1D Schrodinger equation specializing to the linear potential that is perturbed by electric field. The linear and square Stark effects are perturbation terms. Finally, the CFM results will be compared with the exact results. The CFM method is superior to many standard techniques that have been used to solve the Schrodinger equation such as Numervo.
\end{abstract}

Keywords Linear potential $\cdot$ Stark effect $\cdot$ Canonical function method

\section{Introduction}

In quantum mechanics, the analysis of systems with various potentials is important. A system with a symmetrical linear potential $V(z)=F|z|$ is a simple model to analyze neutron quantized balances in the gravity field of earth. A particle with this potential is studied as "a quantum bouncing ball" in [4-6].

L. F. Matin · H. H. Bouzari ( $ه)$

Azad University, Tehran, Iran

e-mail: hh.bouzari@yahoo.com

L. F. Matin

e-mail: laleh.matin@gmail.com

F. Ahmadi

Shahid Rajaee Teacher Training University, Tehran, Iran

e-mail: fahmadi@srttu.edu
The Canonical Function Method (CFM) can handle a large variety of quantum problems [1], also the eigenvalues problem making it an extremely versatile, fast and highly accurate.

The CFM turns the two-point boundary value Schrodinger problem into initial value of Schrodinger problem and allows full and accurate determination of the spectrum. This is done by expressing the solution as a sum of two linearly independent functions (the canonical functions) with specific values at some arbitrary point belonging to the interval defined by the two boundaries. The integration proceeds simultaneously from this point toward the left and right boundaries evaluating at each step a corresponding ratio. It stops when the difference between the left and right ratio is below a given desired precision [2].

In this work, we discuss the application of CFM to a system with a linear potential; we consider the Stark effect as a perturbation term of desired potential (the second-order shifts in the energy spectrum due to an external constant force) in a one-dimensional model quantum mechanical system described by the linear potentials, the so-called quantum bouncer (defined by $V(z)=F z$ for $z>0$ and $V(z)=\infty$ for $z<0$ ) and the symmetric linear potential (given by $V(z)=F|z|)$ ). We show how straightforward use of the most obvious properties of the Airy function solutions gives closed form results for the Stark shifts in this system [3].

This article is organized as follows: the next section is a description of the CFM and exact solutions of Schrodinger Equation with the Linear Potential in 1D with the appropriate boundary conditions. In Sect. 3, the Stark effect for 1D quantum mechanical system described by the linear potentials and the CFM is applied to the Stark effect in the quantum bouncer and the CFM results will be compared with analytical solution of the Stark effect in quantum bouncer (Airy functions). Finally, Sect. 4 bears our conclusions. 


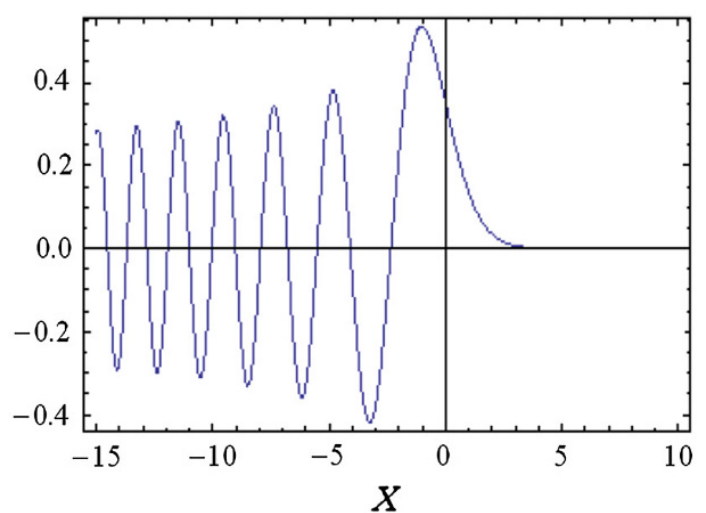

Fig. 1 Plot of eigenfunction $A i(x)$

\section{Solving Schrodinger equation with linear potentials}

Terrestrial objects are confined by the Earth's gravitational field. Since the gravitational interaction is weak, the quantum effects of gravity are not observed in the macroworld. Evidence for the quantized gravitational energy levels is the quantum bouncer (to a so-called "The quantum bouncer") [4]. It has been a favorite example in the repertoire of solvable one-dimensional problem for those who teach quantum physics. This academic problem has received renewed attention as the simplest model for quantum states of neutrons in the Earth's gravitational field [5].

Now, if we consider the Schrodinger equation for a particle under the influence of gravity which is bounced on a perfectly reflecting surface, the particle has this linear potential

$V(z)=\left\{\begin{array}{cc}F Z & Z \geq 0 \\ \infty & Z<0\end{array}\right.$

This problem has been solved by Airy special functions $\operatorname{Ai}(x)$ and $\operatorname{Bi}(x)$ [6]. The Airy functions are solutions of the differential Equation [7]:

$\frac{-\hbar^{2}}{2 m} \frac{d^{2} \psi_{n}(z)}{d z^{2}}+F z \psi_{n}(z)=E_{n} \psi_{n}(z)$

The Airy functions are the solutions of the Schrodinger equation for a particle confined by a triangular well and for a particle in a one-dimensional constant force field. Figure (1) shows $\operatorname{Ai}(x)$ and $\operatorname{Bi}(x)$ [where $\operatorname{Ai}(x)$ and $\operatorname{Bi}(x)$ are the Airy functions].

The exact solution

The Schrodinger equation for the quantum bouncer is:

$\psi_{n}^{\prime \prime}(x)=\left(x-\zeta_{n}\right) \psi_{n}(x)$

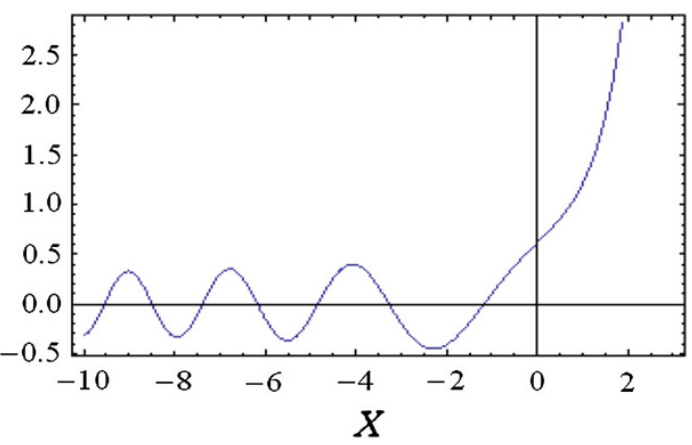

Fig. 2 Plot of eigenfunction $B i(x)$

Using the change of variable and the definitions:

$\zeta_{n}=\frac{E_{n}}{F \rho} \equiv \frac{E_{n}}{\varepsilon_{0}}, z=\rho x, \rho=\left(\frac{\hbar^{2}}{2 m F}\right)^{1 / 3}$

The solutions of Eq. (3) are two linearly independent Airy functions $\operatorname{Ai}\left(x-\zeta_{n}\right)$ and $\operatorname{Bi}\left(x-\zeta_{n}\right)$. The $\operatorname{Bi}\left(x-\zeta_{n}\right)$ solution diverges for large positive argument and does not satisfy the boundary condition $\psi(z \rightarrow \infty)=0$, therefore it is excluded. The energy eigenvalues are determined by the boundary condition imposed by the infinite wall at the origin, namely that $\psi(z=0)=\operatorname{Ai}\left(-\zeta_{n}\right)=0$ (Fig. 2).

The quantized energies are then given in terms of the zeros of the well-behaved Airy function $\operatorname{Ai}\left(-\zeta_{n}\right)$ with $E_{n}=\varepsilon_{0} \zeta_{n}$. The quantized energies become

$$
\begin{aligned}
E_{n}= & \varepsilon_{0}\left(\frac{3 \pi}{8}(4 n-1)\right)^{2 / 3}\left[1+\frac{5}{48}\left(\frac{3 \pi}{8}(4 n-1)\right)^{-2}\right. \\
& \left.-\frac{5}{36}\left(\frac{3 \pi}{8}(4 n-1)\right)^{-4}\right]
\end{aligned}
$$

The eigenfunctions of this potential are:

$\psi_{n}(z)=N_{n} \operatorname{Ai}\left(\frac{z}{\rho}-\zeta_{n}\right), N_{n}=\frac{1}{\sqrt{\rho} \mathrm{A}^{\prime} \mathrm{i}\left(-\zeta_{n}\right)}$

$\mathrm{A}^{\prime} \mathrm{i}\left(-\zeta_{n}\right)$ is differential of $\mathrm{Ai}\left(-\zeta_{n}\right)$ where $\left|\psi_{n}(z)\right|^{2}$ is the probability distribution for a particle that bounces near the surface. All the eigenfunctions for this problem are pieces of the same function, the Airy function Ai, shifted in each case so that it has a zero at $\mathrm{z}=0$ and by $z<0$ part truncated. In Table 1, eigenvalues of the linear potential for the first five levels are illustrated.

\section{The CFM solution}

For solving by CFM method, we start from a point $z_{0} \in$ $\left[z_{1}, z_{2}\right]$ and indicate the solution as a superposition of two linearly independent functions $\alpha(E, z), \beta(E, z)$ depending on the energy $E$ and the abscissa $z$ such that [7]: 
Table 1 Eigenvalues of the levels linear potential for the first five

\begin{tabular}{ll}
\hline$n$ & $E$ (Joule) \\
\hline 1 & $2.33(-32)$ \\
2 & $4.08(-32)$ \\
3 & $5.52(-32)$ \\
4 & $6.78(-32)$ \\
5 & $7.94(-32)$ \\
\hline
\end{tabular}

$\psi(z)=\psi\left(z_{0}\right) \alpha(E, z)+\psi^{\prime}\left(z_{0}\right) \beta(E, z)$

And its derivative is:

$\psi^{\prime}(z)=\psi\left(z_{0}\right) \alpha^{\prime}(E, z)+\psi^{\prime}\left(z_{0}\right) \beta^{\prime}(E, z)$

Two linear independent functions $\alpha(\mathrm{E}, \mathrm{z}), \beta(\mathrm{E}, \mathrm{z})$ are:

$\left\{\begin{array}{l}\alpha(E, z)=A \psi_{1}(z)+B \psi_{2}(z) \\ \beta(E, z)=C \psi_{1}(z)+D \psi_{2}(z)\end{array}\right.$

The CFM is based on the extraction of eigenvalues from the zeros of the eigenvalues function $F(E)$ which is defined from the solution of the left $\left(z \rightarrow z_{1}\right)$ and right $\left(z \rightarrow z_{2}\right)$. Functions $1_{+}(E)$ and $1_{-}(E)$ by the ratio of canonical functions $\alpha(E, z), \beta(E, z)$ or their derivatives are obtained.

$F(E)=1_{+}(E)-1_{-}(E)=\left[\frac{\psi^{\prime}\left(z_{0}\right)}{\psi\left(z_{0}\right)}\right]_{+}-\left[\frac{\psi^{\prime}\left(z_{0}\right)}{\psi\left(z_{0}\right)}\right]_{-}$

There are several types of the eigenvalues functions defined as the differences between left and right ratio function [7].

The initial condition could be used in this problem:

$\psi\left(z_{1}\right)=\psi\left(z_{2}\right)=0$

The functions $1_{+}(E), 1_{-}(E)$ are:

$1_{-}(E)=\lim _{z \rightarrow z_{1}}-\frac{\alpha(E, z)}{\beta(E, z)}$

$1_{+}(E)=\lim _{z \rightarrow z_{2}}-\frac{\alpha(E, z)}{\beta(E, z)}$

Equations (9) are simplified to:

$\alpha(E, z)=A\left\{\psi_{1}(z)-\frac{\psi_{1}^{\prime}\left(z_{0}\right)}{\psi_{2}^{\prime}\left(z_{0}\right)} \psi_{2}(z)\right\}$

$\beta(E, z)=A\left\{\psi_{1}(z)-\frac{\psi_{1}\left(z_{0}\right)}{\psi_{2}\left(z_{0}\right)} \psi_{2}(z)\right\}$

By considering to Eqs. (10), (11) and (12), we get to:

$F(E)=\frac{A\left\{\psi_{1}\left(z_{1}\right)-\frac{\psi_{1}^{\prime}\left(z_{0}\right)}{\psi_{2}^{\prime}\left(z_{0}\right)} \psi_{2}\left(z_{1}\right)\right\}}{C\left\{\psi_{1}\left(z_{1}\right)-\frac{\psi_{1}\left(z_{0}\right)}{\psi_{2}\left(z_{0}\right)} \psi_{2}\left(z_{1}\right)\right\}}-\frac{A\left\{\psi_{1}\left(z_{2}\right)-\frac{\psi_{1}^{\prime}\left(z_{0}\right)}{\psi_{2}^{\prime}\left(z_{0}\right)} \psi_{2}\left(z_{2}\right)\right\}}{C\left\{\psi_{1}\left(z_{2}\right)-\frac{\psi_{1}\left(z_{0}\right)}{\psi_{2}\left(z_{0}\right)} \psi_{2}\left(z_{2}\right)\right\}}$

$=0$
Table 2 The first five levels of the eigenvalues of the linear potential are given by the CFM method exact results

\begin{tabular}{lll}
\hline $\begin{array}{l}\text { Level of } \\
\text { energy }\end{array}$ & $E$ (Joule) CFM results & $E($ Joule) Exact results \\
\hline 1 & $2.3381074104597674(-32)$ & $2.3381074104597674(-32)$ \\
2 & $4.087949444130971(-32)$ & $4.087949444130970(-32)$ \\
3 & $5.520559828095551(-32)$ & $5.520559828095552(-32)$ \\
4 & $6.7867080900717600(-32)$ & $6.786708090071759(-32)$ \\
5 & $7.944133587120853(-32)$ & $7.944133587120853(-32)$ \\
\hline
\end{tabular}

By simplification:

$\left\{\frac{\psi_{1}\left(z_{0}\right)}{\psi_{2}\left(z_{0}\right)}-\frac{\psi_{1}^{\prime}\left(z_{0}\right)}{\psi_{2}^{\prime}\left(z_{0}\right)}\right\} \times\left[\psi_{2}\left(z_{1}\right) \psi_{1}\left(z_{2}\right)-\psi_{1}\left(z_{1}\right) \psi_{2}\left(z_{2}\right)\right]=0$

Equation (13) has two factors, one of them contains only values at $z=z_{0}$ and the other contains values $z_{1}$ and $z_{2}$ that we should put it equal to zero:

$\psi_{2}\left(z_{1}\right) \psi_{1}\left(z_{2}\right)-\psi_{1}\left(z_{1}\right) \psi_{2}\left(z_{2}\right)=0$

The eigenfunctions of the linear potential (6) are:

$\psi_{n}(z)=N_{n} A i\left(\frac{z}{\rho}-\zeta_{n}\right), N_{n}=\frac{1}{\sqrt{\rho} A^{\prime} i\left(-\zeta_{n}\right)}$

Therefore, Eq. (14) becomes:

$\left[\operatorname{Bi}\left(z_{1}-\zeta_{n}\right) \operatorname{Ai}\left(z_{2}-\zeta_{n}\right)-\operatorname{Ai}\left(z_{1}-\zeta_{n}\right) \operatorname{Bi}\left(z_{2}-\zeta_{n}\right)\right]=0$

Equation (15) is an equation from $\zeta_{n}\left(E_{n}\right), z_{1}$ and $z_{2}$ where $\zeta_{n}\left(E_{n}\right)$ is unknown, but $z_{1}$ and $z_{2}$ are known and arbitrary (Table 2).

The aim is to find those values of $E_{n}$ for which the left side of (15) vanished.

By solving Eq. (15), we get Table 1. (Supposed $z_{1}=0, z_{2}=20$ ). To find the zeros of Eq. (15), we have used "Newton method" in Mathematica by "Find Root" method as a numerical method.

The results of the CFM method are so near to the exact results, it means that this method is so useful and accurate; the results of CFM method in all of the levels of energies are so near to the exact result.

\section{The Stark effect in linear potentials}

Consider a particle (electron) is subject to the influence of gravity $(\mathrm{g})$ and electric field $\epsilon_{0}$. In this case, the level of energy is shifted due to this electric field (Table 3). 
Table 3 Shift of first-order energy (linear stark effect)

Hamiltonian of particle in the gravity of earth is:

$H_{0}=\frac{p^{2}}{2 m}+m g z$

Considering first-and second-order stark effect:

$H=H_{0}+H_{1}=\frac{p^{2}}{2 m}+m g z+e \epsilon_{0} z+\frac{1}{2} e^{2} \epsilon_{0}^{2} z^{2}$

The Exact solution

On the basis of Eq. (17), the Schrodinger equation is written as follows:

$\frac{-\hbar^{2}}{2 m} \frac{\mathrm{d}^{2}}{\mathrm{~d} z^{2}} \psi_{n}(z)=\left(-\frac{1}{2} e^{2} \epsilon_{0}^{2} z^{2}-\left(m g+e \epsilon_{0}\right) z+E_{n}\right) \psi_{n}(z)$

Equation (18) can be written as follows:

That can be written as:

$\psi_{n}^{\prime \prime}(z)-\left(a z^{2}+b z-c\right) \psi_{n}(z)=0$

Where

$a=\frac{m e^{2} \in_{0}^{2}}{\hbar^{2}}, b=\frac{2 m}{\hbar^{2}}\left(m g+e \epsilon_{0}\right), c=\frac{2 m}{\hbar^{2}} E_{n}$

Using the change of variables:

$\xi=z+b / 2 a, u=\sqrt{4} a \xi$

Equation (19) becomes:

$\frac{\mathrm{d}^{2} \psi}{\mathrm{d} u^{2}}+\left(\frac{c-\frac{b^{2}}{4 a}}{\sqrt{a}}-u^{2}\right) \psi(u)=0$

The harmonic oscillator Schrodinger's equation is [8]:

$\psi^{\prime \prime}(z)+\left(2 n+1-z^{2}\right) \psi(z)=0$

Where value of energies is:

$\frac{2 E}{\hbar w}=2 n+1$

$\mathrm{Or}$

$E_{n}=\left(n+\frac{1}{2}\right) \hbar w ; \quad n=0,1,2, \ldots$

Comparison of Eq. (22), (23) and considering the boundary condition $\psi(z \rightarrow \infty)=0$, we have: $c=(2 n+1) \sqrt{a}+\frac{b^{2}}{4 a}$

Substituting Eq. (20) in Eq. (26), the exact eigenvalues are:

$E_{n}=\left(n+\frac{1}{2}\right) \frac{e \epsilon_{0} \hbar}{\sqrt{m}}+\frac{\left(m g+e \epsilon_{0}\right)^{2}}{2 e^{2} \epsilon_{0}^{2}}$

Supposing that $n=1, \epsilon_{0}=10^{-11 \mathrm{~V}} / \mathrm{m}$ and $\hbar=1.054 \times$ $10^{-34}$ j.s, $m_{e}=9.11 \times 10^{-31} \mathrm{~kg}, e=1.6 \times 10^{-19} \mathrm{C}, g=$ $9.8 \mathrm{~m} / \mathrm{s}^{2}$.

Exact value of first level of energy becomes:

$E=0.81 \times 10^{-48} \mathrm{j}$

The Perturbed solution

According to the Subsect. (2.1), the normalized eigenfunctions of the unperturbed linear potential is:

$\psi_{n}(z)=N_{n} \mathrm{Ai}\left(\frac{z}{\rho}-\zeta_{n}\right), N_{n}=\frac{1}{\sqrt{\rho} \mathrm{A}^{\prime} \mathrm{i}\left(-\zeta_{n}\right)}$

By assuming linear stark effect, the Perturbed Hamiltonian becomes:

$H=H_{0}+H_{1}=\frac{p^{2}}{2 m}+m g z+e \in_{0} z$

The first-order energy shift is:

$\Delta E_{n}^{(1)}=\left\langle\psi_{n}\left|H_{1}\right| \psi_{n}\right\rangle$

By replacing the perturbed potential, we have [8]:

$\Delta E_{n}^{(1)}=e \in_{0} \int_{0}^{\infty} \frac{z d z}{\rho\left[\mathrm{A}^{\prime} \mathrm{i}\left(-\zeta_{n}\right)\right]^{2}} \operatorname{Ai}\left(\frac{z}{\rho}-\zeta_{n}\right)^{2}$

Or

$\Delta E_{n}^{(1)}=e \in_{0} \rho\left(\frac{2}{3} \zeta_{n}\right)=\frac{2}{3}\left(\frac{e \epsilon_{0}}{m g}\right) \varepsilon_{0} \zeta_{n}=\frac{2}{3}\left(\frac{e \epsilon_{0}}{m g}\right) E_{n}$

Now, considering the square stark effect, Hamiltonian becomes:

$H=\frac{p^{2}}{2 m}+m g z+e \in_{0} z+\frac{1}{2} e^{2} \in_{0}^{2} z^{2}$

Then, the perturbed potential caused by the square stark effect is:

$V=\frac{1}{2} e^{2} \in_{0}^{2} z^{2}$

Hence, the shift of energy is:

$\Delta E_{n}^{(1)}=\left\langle\psi_{n}\left|\frac{1}{2} e^{2} \in_{0}^{2} z^{2}\right| \psi_{n}\right\rangle$ 
Or

$\Delta E_{n}^{(1)}=\frac{1}{2} e^{2} \in_{0}^{2} \int_{0}^{\infty} \frac{z^{2} d z}{\rho\left[\mathrm{A}^{\prime} \mathrm{i}\left(-\zeta_{n}\right)\right]^{2}} \mathrm{Ai}\left(\frac{z}{\rho}-\zeta_{n}\right)^{2}$

So, Eq. (36) reduces to:

$$
\begin{aligned}
\Delta E_{n}^{(1)} & =-\frac{8}{30} e^{2} \in_{0}^{2} \zeta_{n}^{2} \rho^{2}=-\left(e^{2} \in_{0}^{2} \rho^{2}\right)\left(\frac{8}{30} \zeta_{n}^{2}\right) \\
& =-\frac{8}{30}\left(\frac{e \epsilon_{0}}{m g}\right)^{2} E_{n}
\end{aligned}
$$

where $\epsilon_{0}=10^{-11} \mathrm{v} / \mathrm{m}, m_{e}=9.11 \times 10^{-31} \mathrm{~kg}, \quad e=1.6 \times$ $10^{-19} \mathrm{C}, g=9.8^{\mathrm{m}} / \mathrm{s}^{2}$.

Table 4 illustrates shift of energy by the square stark effect and Table 5 compares influence of the linear and square stark effect on shift of energy.

\section{The CFM Solution}

To apply the CFM method, first we note that the exact eigenfunction of harmonic oscillator with boundary condition $\psi(\mathrm{z} \rightarrow \pm \infty)=0$, is as:

$\psi_{n}(z)=y e^{-z^{2} / 2}$

where $y$ is Hermite function. The Hermite functions satisfy the differential Eq. (23).

Therefore, the eigenfunction can be defined as follows [9]:

$\psi_{n}(z)=\left(2^{2} n ! \sqrt{\pi}\right) e^{-z^{2} / 2} H_{n}(z),-\infty<z<+\infty$

Or

$\psi_{n}(z)=\left(\frac{2^{2} n ! \sqrt{\pi}}{2}\right) e^{-z^{2} / 2} H_{n}(z), 0<z<+\infty$

Table 4 Shift of energy (square stark effect)

\begin{tabular}{ll}
\hline$n$ & $\Delta E($ Joule $)$ \\
\hline 1 & $-0.019(-32)$ \\
2 & $-0.034(-32)$ \\
3 & $-0.051(-32)$ \\
4 & $-0.057(-32)$ \\
5 & $-0.067(-32)$ \\
\hline
\end{tabular}

where Hermite functions, $\mathrm{H}_{\mathrm{n}}(\mathrm{z})$, can be defined in terms of the polynomials [9]:

$y_{1}(z)=1-\frac{2 n}{2 !} z^{2}+\frac{2^{2} n(n-2)}{4 !} z^{4}-\frac{2^{3} n(n-2)(n-4)}{4 !} z^{6}+\ldots$

$n=0,2,4, \ldots$

$$
\begin{aligned}
y_{2}(z)= & z-\frac{2 n(n-1)}{3 !} z^{3}+\frac{2^{2}(n-1)(n-3)}{5 !} z^{5} \\
& -\frac{2^{3}(n-1)(n-3)(n-5)}{7 !} z^{7}+\ldots
\end{aligned}
$$

$n=1,3,5, \ldots$

That $y_{1}(z), y_{2}(z)$ are convergent for all of $z$.

As was determined in Sect. 2.2 by considering relations (7-15) and (40), the Eq. (14) equals to:

$H_{1}\left(u_{2}\right) H_{2}\left(u_{1}\right)-H_{1}\left(u_{1}\right) H_{2}\left(u_{2}\right)=0$

Therefore, by supposing initial conditions $z_{1}=0$ and $z_{2}=1$, we have:

$u_{1}=b / 2 a^{3 / 4}, u_{2}=\sqrt{4} a(1+b / 2 a)$

So Eq. (43) equals to:

$H_{1}(\sqrt{4} a(1+b / 2 a)) H_{2}\left(b / 2 a^{3 / 4}\right)-H_{1}\left(b / 2 a^{3 / 4}\right)$

$H_{2}(\sqrt{4} a(1+b / 2 a))=0$

By considering values $a, b, c$ (20) and Eqs. (41) and (42), we obtain:

$H_{1}\left(b / 2 a^{3 / 4}\right)=1, H_{2}\left(b / 2 a^{3 / 4}\right)=0$

Then, Eq. (44) reduces to:

$H_{2}(\sqrt{4} a(1+b / 2 a))=0$

Substituting the first three terms of Eq. (42) into Eq. (45) yields:

$$
\begin{gathered}
\sqrt{4} a\left(1+\frac{b}{2 a}\right)-\frac{2(n-1)}{3 !}\left(\sqrt{4} a\left(1+\frac{b}{2 a}\right)\right)^{3} \\
+\frac{2^{2}(n-1)(n-3)}{5 !}\left(\sqrt{4} a\left(1+\frac{b}{2 a}\right)\right)^{5}=0
\end{gathered}
$$

Table 5 Illustration and comparison of the first five levels of unperturbed linear potential and linear stark effect and square stark effect

\begin{tabular}{llll}
\hline$n$ & $E($ Joule)Linear potential & $\Delta E($ Joule)Linear stark effect & $\Delta E($ Joule)square stark effect \\
\hline 1 & $2.33(-32)$ & $0.27(-32)$ & $-0.019(-32)$ \\
2 & $4.08(-32)$ & $0.44(-32)$ & $-0.034(-32)$ \\
3 & $5.52(-32)$ & $0.60(-32)$ & $-0.051(-32)$ \\
4 & $6.78(-32)$ & $0.74(-32)$ & $-0.057(-32)$ \\
5 & $7.94(-32)$ & $0.84(-32)$ & $-0.067(-32)$ \\
\hline
\end{tabular}


Equation (46) can be rewritten as follows:

$$
\begin{aligned}
1 & -\frac{2(n-1)}{3 !}\left(\sqrt{4} a\left(1+\frac{b}{2 a}\right)\right)^{2}+\frac{2^{2}(n-1)(n-3)}{5 !} \\
& \times\left(\sqrt{4} a\left(1+\frac{b}{2 a}\right)\right)^{4}=0
\end{aligned}
$$

To find the zeros of Eq. (47), we have used instruction of "Find Root" method in Mathematica software such as a numerical method. Therefore, we obtain:

$n=1.7 \times 10^{10}$

By replacing value of $\mathrm{n}$ in Eq. (26) and considering values of $\hbar, m, e, g$, the energy equals:

$E=0.18 \times 10^{-37} \mathrm{j}$

\section{Conclusion}

In this paper, the perturbed linear potentials due to influence of electrical field were studied. The stark effect in the linear potential was investigated in three methods (Exact, CFM and Perturbed). We show that the Airy functions $(\operatorname{Ai}(x), \operatorname{Bi}(x))$ are eigenfunctions of the unperturbed linear potential. Since the $\operatorname{Bi}(x)$ solution diverges for large positive argument and it does not satisfy the boundary condition $\psi(\infty)=0$, therefore it is excluded. Then, the quantized energies are then given in terms of the zeros of the well-behaved Airy function $\operatorname{Ai}\left(-\zeta_{n}\right)$ with $E_{n}=\varepsilon_{0} \zeta_{n}$.

Also, we investigated the stark effect in the linear potential that is term of perturbation in the Schrodinger equation. The exact solution leads to Hermite functions and eigenvalues adherence relation of $E_{n}=\left(n+\frac{1}{2}\right) \frac{e \epsilon_{0} \hbar}{\sqrt{m}}+\frac{\left(m g+e \epsilon_{0}\right)^{2}}{2 e^{2} \epsilon_{0}^{2}}$.
Finally, the eigenvalues were obtained by the perturbed theory and CFM method. The eigenvalues of the system by CFM method are so near to the exact results but the consequence of perturbation predictions does not have this precision.

Acknowledgments This work was supported by the Islamic Azad University (IAU), North Tehran Branch.

Authors' contributions The author did not provide this information.

Conflict of interest The author did not provide this information.

Open Access This article is distributed under the terms of the Creative Commons Attribution License which permits any use, distribution, and reproduction in any medium, provided the original author(s) and the source are credited.

\section{References}

1. Tannous, C., Fakhreddine, K., Langlois, J.: Phys. Rep. 467, 173 (2008)

2. Tannous,C., Langlois, J. arXiv: 1003.0184v1 [quant-ph] 28 Feb 2010

3. Robinett, RW. arXiv: 0909.2209v1 [quant-ph] 11 Sep 2009

4. Gibbs, R.L.: The quantum bouncer. Am. J. Phys. 43, 25-28 (1975)

5. Nesvizhevsky, V.V., et al.: Quantum states of neutrons in the earth's gravitational field. Nature 415, 297-306 (2002)

6. Vallee, O., Soares, M: Airy Functions and Applications to Physics. 137-142

7. Tannous, C., Fakhreddine, K., Langlois, J. arXiv: quant- ph/ 0702110v1, 12 Feb 2007 and arXiv: quant-ph (2010)

8. Robinett, RW. The Stark effect in linear potentials, Eur. J. Phys. 31. 78-89, arXiv: quant-ph (2010)

9. George, F. Simmons by MC Grew, differentials equations with application and historical notes (1972) 